\title{
Introduction to the special issue on therapeutic ultrasound
}

\author{
Vera Khokhlova and Lawrence Crum \\ Moscow State University, Leninskie Gory, Moscow, 119991, Russia
}

(Received 24 June 2013; accepted 24 June 2013)

[http://dx.doi.org/10.1121/1.4813105]

PACS number(s): 43.80.Sh, 43.80.Ev, 43.80.Vj, 43.80.Gx [ADP]

In the 1950s, the brothers William and Francis Fry treated a number of patients for Parkinson's disease by focusing high intensity ultrasound on the brains of patients after some part their skull was removed. Local thermal ablations were created in targeted areas of brain and these treatments were shown to be beneficial to the patients. At the same time, in the former USSR, the team of A.K. Burov used high intensity unfocused ultrasound to treat aggressive melanoma cancerous tumors and obtained very promising positive response in a number of patients; similar exposure protocols were used to induce specific immune response in an animal model.

However, in those days, imaging systems were in their infancy, no MRI or CT systems were available, and ultrasound technology was not ready to extensively explore the ability of ultrasound to non-invasively induce changes in biological tissue. The advancements in tissue imaging, engineering, and the rapid development of computers that occurred during the last decade have now permitted a new cycle of development of therapeutic ultrasound. Successful medical technologies are already in clinical practice, ranging from tumor treatments to cosmetic applications; clinical studies are under way in ultrasound enhanced drug delivery, sonothrombolysis, and hyperthermia. Recently, a pilot study of the use of focused ultrasound for the treatment of dyskinesia in Parkinson's disease was funded by the Michael J. Fox Foundation bringing back Fry's efforts at a new technological level. Every large acoustics professional meeting, and in particular ASA meetings, include special sessions on therapeutic ultrasound. The annual International Symposium for Therapeutic Ultrasound and the biannual Focused Ultrasound Surgery Foundation Symposium bring world experts together to exchange ideas and accelerate development of this field.

Even though therapeutic ultrasound technology is rapidly evolving into clinical practice, basic acoustics still remains an important foundation and a critical component in its successful implementation. This issue represents only a snapshot of the scientific and clinical applications at this moment of time. Many questions are not yet answered. Further progress of the field requires merging new interesting acoustic phenomena with biophysics, engineering solutions, and other exciting interdisciplinary studies. We hope that some of the examples of recent studies presented in this issue will be of general interest to the acoustical community helping to bring a younger generation of acousticians into the field. We also anticipate that some of the results reported in this issue will soon be transitioned into the clinic, resulting in the improvement of health care for our society. 